\title{
Buildings with isolated subspaces and relatively hyperbolic Coxeter groups
}

\author{
Pierre-Emmanuel Caprace*
}

\begin{abstract}
Let $(W, S)$ be a Coxeter system. We give necessary and sufficient conditions on the Coxeter diagram of $(W, S)$ for $W$ to be relatively hyperbolic with respect to a collection of finitely generated subgroups. The peripheral subgroups are necessarily parabolic subgroups (in the sense of Coxeter group theory). As an application, we present a criterion for the maximal flats of the Davis complex of $(W, S)$ to be isolated. If this is the case, then the maximal affine sub-buildings of any building of type $(W, S)$ are isolated.
\end{abstract}

Keywords: Coxeter group, building, isolated flat, relative hyperbolicity

MSC 2000: 20F67; 20E42, 20F55, 20F69

\section{Introduction}

Let $X$ be a complete CAT(0) space. A $k$-flat in $X$ is a subset which is isometric to the $k$-dimensional Euclidean space. Since we will mainly be interested in isolated flats, it is convenient to define a flat as a $k$-flat for some $k \geq 2$. In particular, geodesic lines are not considered to be flats. Let $\mathscr{F}$ be a collection of closed convex subsets of $X$. We say that the elements of $\mathscr{F}$ are isolated in $X$ if the following conditions hold:

(A) There is a constant $D<\infty$ such that each flat $F$ of $X$ lies in a tubular $D$-neighbourhood of some $C \in \mathscr{F}$.

\footnotetext{
*F.N.R.S. research associate. This work was partially supported by IPDE.
} 



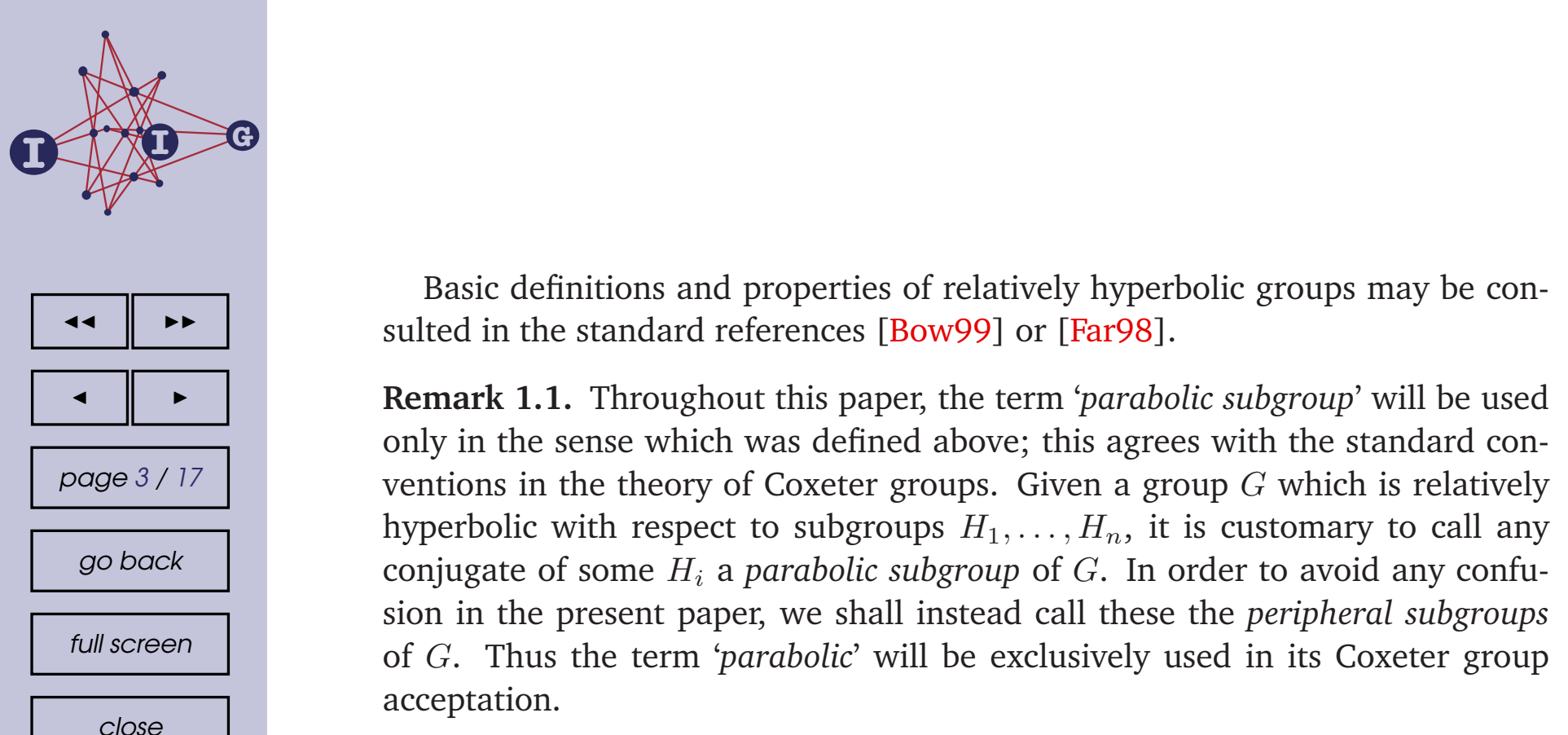

Notice that we do not assume the buildings to be locally compact in (iv). Conditions (RH1) and (RH2) can be checked concretely on the Coxeter diagram of $(W, S)$. Combining Theorem A with the following, one obtains in particular a complete characterization of those Coxeter groups which are relatively hyperbolic with respect to any family of finitely generated subgroups:

Theorem B. Let $(W, S)$ be a Coxeter system with $S$ finite. If $W$ is relatively hyperbolic with respect to finitely generated subgroups $H_{1}, \ldots, H_{m}$, then each $H_{i}$ is a parabolic subgroup of $W$.

It should be noted that there exist non-affine Coxeter groups which are not relatively hyperbolic with respect to any family of parabolic subgroups. Consider, for example, the Coxeter group $W$ with Coxeter generating set $S=$ $\left\{s_{1}, \ldots, s_{n}\right\}$ defined by the following relations: $\left[s_{i}, s_{j}\right]=1$ for $|i-j| \geq 2$ and $o\left(s_{i} s_{j}\right)=4$ for $|i-j|=1$. It is easily verified, using Theorem A, that for $n>7$, the group $W$ is not relatively hyperbolic with respect to any collection of proper parabolic subgroups. For $n=7$, one checks that the set

$$
\mathscr{T}=\{\{1,2,3,5,6,7\},\{2,3,4\},\{3,4,5\},\{4,5,6\}\}
$$

satisfies (RH1) and (RH2).

It happens however quite often that a Coxeter group is relatively hyperbolic with respect to a maximal proper parabolic subgroup:

Corollary C. Suppose that there exists an element $s_{0} \in S$ such that $\left\{s_{0}\right\}^{\perp}$ is spherical. Then $W$ is relatively hyperbolic with respect to the parabolic subgroups whose type belongs to the set

$$
\mathscr{T}=\left\{S \backslash\left\{s_{0}\right\}\right\} \cup\left\{J \subset S \mid J \text { is affine and contains } s_{0}\right\} .
$$

Of particular interest is the special case when the peripheral subgroups of a relatively hyperbolic group are virtually nilpotent (or more generally amenable). 


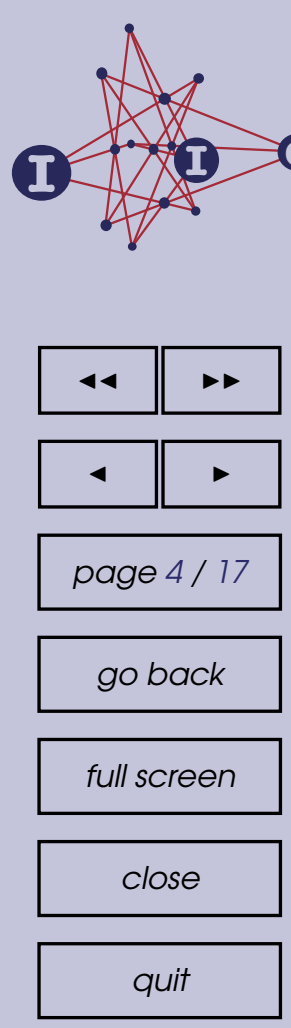

As for any discrete group acting properly and cocompactly on a CAT(0) space, any amenable subgroup of a Coxeter group is virtually abelian. Theorem A yields the following characterization:

Corollary D. The following assertions are equivalent:

(i) For all non-spherical $J_{1}, J_{2} \subset S$ such that $\left[J_{1}, J_{2}\right]=1$, the group $\left\langle J_{1} \cup J_{2}\right\rangle$ is virtually abelian.

(ii) For each minimal hyperbolic $J \subset S$, the set $J^{\perp}$ is spherical.

(iii) The Davis complex $X(W, S)$ has isolated flats.

(iv) The group $W$ is relatively hyperbolic with respect to a collection of virtually abelian subgroups of rank at least 2.

(v) In any building of type $(W, S)$, the maximal residues of (non-spherical) Euclidean type (i.e. the maximal non-spherical Euclidean sub-buildings) are isolated.

The list of all minimal hyperbolic Coxeter systems is known (see [Bou68, Ch. V, pp.133-134] or [Hum90, II.6.9]); in fact, it is not difficult to see that a minimal hyperbolic subset of $S$ has cardinality at most 10. Many Coxeter systems $(W, S)$ such that $W$ is not Gromov hyperbolic do satisfy condition (ii). In particular $S$ may contain affine subsets.

Theorem A is deduced from the detailed study of flats in buildings and Coxeter groups which is made in [CH06]. The equivalence between (ii) and (iii) is a consequence of [HK05, Appendix]. In fact, the theorem above allows one to apply varied algebraic and geometric consequences of the isolation of subspaces established in [loc. cit.] and [DS05]. We collect a few of them in the special case of virtually abelian peripheral subgroups:

Corollary E. Assume that $(W, S)$ satisfies the equivalent conditions of Corollary D. Let $Y$ be a building of type $(W, S), \mathscr{F}$ be the collection of maximal residues of non-spherical Euclidean type and $\Gamma<\operatorname{Isom}(Y)$ be a subgroup acting properly discontinuously and cocompactly. Then:

(i) $\Gamma$ is relatively hyperbolic with respect to the family of stabilizers of elements of $\mathscr{F}$; each of these stabilizers is a cocompact lattice of a Euclidean building.

(ii) $W$ and $\Gamma$ are biautomatic.

(iii) Every connected component of $\partial_{T} X(W, S)$ (resp. $Y$ ) is either an isolated point or a Euclidean sphere (resp. a spherical building).

(iv) Every asymptotic cone of $X(W, S)$ (resp. $Y$ ) is tree-graded with respect to a family of closed convex subsets which are flats (resp. Euclidean buildings); furthermore any quasi-isometry of $X(W, S)$ (resp. $Y$ ) permutes these pieces. 
We refer to [DS05] for more information on asymptotic cones and treegraded spaces. It is known that all Coxeter groups are automatic [BH93], but the problem of determining which Coxeter groups are biautomatic is still incompletely solved: it follows from [CM05] that $W$ is biautomatic whenever $S$ has no irreducible affine subset of cardinality $\geq 3$. Corollary E shows biautomaticity in many other cases.

Let us finally mention that the construction of cocompact lattices in $\operatorname{Isom}(Y)$ is a delicate problem, unless the Coxeter system $(W, S)$ is right-angled (i.e. $o(s t) \in\{1,2, \infty\}$ for all $s, t \in S)$. This question seems especially interesting when $(W, S)$ is 2 -spherical, namely $o(s t)<\infty$ for all $s, t \in S$. Besides the classical case of Euclidean buildings, some known constructions provide examples of lattices when $W$ is 2-spherical and Gromov hyperbolic [KV06]. However, I don't know any example of a cocompact lattice in $\operatorname{Isom}(Y)$ in the case when $W$ is a 2-spherical Coxeter group which is neither Euclidean nor Gromov-hyperbolic. The situation is completely different when $(W, S)$ is right-angled. In that case indeed, graphs of groups provide a large family of examples of cocompact lattices to which Corollary E may be applied.

In order to state this properly, let $A$ be a finite simple graph with vertex set $I$ and for each $i \in I$, let $P_{i}$ be a group. Let $\Gamma=\Gamma\left(A,\left(P_{i}\right)_{i \in I}\right)$ be the group which is the quotient of the free product of the $\left(P_{i}\right)_{i \in I}$ by the normal subgroup generated by all commutators of the form $\left[g_{i}, g_{j}\right]$ with $g_{i} \in P_{i}, g_{j} \in P_{j}$ and $\{i, j\}$ spanning an edge of $A$. Let also $\left(W,\left\{s_{i}\right\}_{i \in I}\right)$ be the Coxeter system such that $o\left(s_{i} s_{j}\right)=2$ (resp. $o\left(s_{i} s_{j}\right)=\infty$ ) for each edge (resp. non-edge) $\{i, j\}$ of $A$. Then $\Gamma$ acts simply transitively on the chambers of a building $Y\left(A,\left(P_{i}\right)_{i \in I}\right)$ of type $\left(W,\left\{s_{i}\right\}_{i \in I}\right)$ by [Dav98, Theorem 5.1 and Corollary 11.7]. If each $P_{i}$ is finite, then this building is locally compact and, hence, $\Gamma$ is a cocompact lattice in its automorphism group. For example, if the graph $A$ is a $n$-cycle with $n \geq 5$, then this building is a Bourdon building and $\Gamma$ is a Bourdon lattice (these are the Fuchsian buildings and their lattices defined and studied by M. Bourdon in [Bou97]). Moreover, if each $P_{i}$ is infinite cyclic, then $\Gamma$ is a right-angled Artin group.

For the Coxeter system $\left(W,\left\{s_{i}\right\}_{i \in I}\right)$, condition (ii) of Corollary D may be expressed as follows: for each 3-subset $J \subset I$ which is not a triangle, the subgraph induced on $J^{\perp}=\{i \in I \mid\{i, j\}$ is an edge for each $j \in J\}$ is a complete graph. Let now $\mathcal{S}_{\text {aff }}$ be the set of all subsets of $J$ of the form $\left\{i_{1}, j_{1}, \ldots, i_{n}, j_{n}\right\}$ where $\left\{i_{k}, j_{k}\right\}$ is a non-edge for each $k$ but all other pairs of elements are edges. Note that for $J=\left\{i_{1}, j_{1}, \ldots, i_{n}, j_{n}\right\}$, the subgroup $\Gamma_{J}$ of $\Gamma$ generated by all $P_{i}$ 's with $i \in J$ has the following structure:

$$
\Gamma_{J} \simeq\left(P_{i_{1}} * P_{j_{1}}\right) \times \cdots \times\left(P_{i_{n}} * P_{j_{n}}\right) .
$$


Then Corollary E implies that, under the assumption (ii), the group $\Gamma$ is relatively hyperbolic with respect to the family of all conjugates of subgroups of the form $\Gamma_{J}$ with $J \in \mathcal{S}_{\text {aff }}$.

\section{On parabolic subgroups of Coxeter groups}

Recall that a subgroup of $W$ of the form $W_{J}$ for some $J \subset S$ is called a standard parabolic subgroup. Any of its conjugates is called a parabolic subgroup of $W$. A basic fact on Coxeter groups is that any intersection of parabolic subgroups is itself a parabolic subgroup. This allows to define the parabolic closure $\operatorname{Pc}(R)$ of a subset $R \subset W$ : it is the smallest parabolic subgroup of $W$ containing $R$.

Lemma 2.1. Let $G$ be a reflection subgroup of $W$ (i.e. a subgroup of $W$ generated by reflections). Then there is a set of reflections $R \subset G$ such that $(G, R)$ is a Coxeter system. Furthermore, if $(G, R)$ is irreducible (resp. spherical, affine of rank $\geq 3$ ), then so is $\operatorname{Pc}(G)$.

Proof. For the first assertion, see [Deo89]. Any two reflections in $R$ which do not commute lie in the same irreducible component of $\operatorname{Pc}(R)$. Therefore, if $(G, R)$ is irreducible, then all elements of $R$ are in the same irreducible component of $\operatorname{Pc}(R)$. Since $G=\langle R\rangle$ and $\operatorname{Pc}(R)$ is the minimal parabolic subgroup containing $G$, we deduce that $\operatorname{Pc}(R)$ is irreducible. If $G$ is finite, then it is contained in a finite parabolic subgroup (see [Bou68]), hence $\operatorname{Pc}(G)$ is spherical. Finally, if $(G, R)$ is affine of rank $\geq 3$, then so is $\operatorname{Pc}(G)$ by [Cap06, Proposition 16].

Lemma 2.2. Let $P \subset W$ be an infinite irreducible parabolic subgroup. Then the normalizer of $P$ in $W$ splits as a direct product: $\mathscr{N}_{W}(P)=P \times \mathscr{Z}_{W}(P)$ and $\mathscr{Z}_{W}(P)$ is also a parabolic subgroup of $W$.

Proof. See [Deo82, Proposition 5.5].

Lemma 2.3. Let $G_{1}, G_{2}$ be finitely generated reflection subgroups of $W$ which are irreducible, i.e. $\left(G_{i}, R_{i}\right)$ is irreducible for $R_{i} \subset G_{i}$ as in Lemma 2.1, and assume that $G_{1}$ is infinite and that $\left[G_{1}, G_{2}\right]=\{1\}$. Then either

$$
\operatorname{Pc}\left(\left\langle G_{1} \cup G_{2}\right\rangle\right) \simeq \operatorname{Pc}\left(G_{1}\right) \times \operatorname{Pc}\left(G_{2}\right)
$$

or $\operatorname{Pc}\left(G_{1}\right)=\operatorname{Pc}\left(G_{2}\right)$ is an irreducible affine Coxeter group of rank $\geq 3$. 


For assertion (ii), choose $R$ amongst the residues whose stabilizer is $P$ in such a way that it minimizes the distance to $F$. If some point of $F$ does not belong to $R$, there exists a wall which separates that point from its projection to $R$. By (i) this walls separates $F$ from $R$ and is perpendicular to every wall of $R$. Transforming $R$ by the reflection through that wall, we obtain another residue whose stabilizer is $P$, but closer to $F$. This contradicts the minimality assumption made on $R$.

\section{Relative hyperbolicity}

\subsection{Peripheral subgroups are parabolic}

The purpose of this section is to prove Theorem B. We will need a subsidiary result on Coxeter groups. In order to state it properly, we make use of some additional terminology which we now introduce.

Given an element $w \in W$ and a half-space $\mathcal{H}$ of the Cayley graph $\operatorname{Cay}(W, S)$ (or of the Davis complex $X(W, S)$ ), we say that $\mathcal{H}$ is $w$-essential if $w . \mathcal{H} \subsetneq \mathcal{H}$ or $w^{-1} . \mathcal{H} \subsetneq \mathcal{H}$. Notice that an element $w \in W$ admits a $w$-essential half-space if and only if it has infinite order.

The reflection of $W$ associated to $\mathcal{H}$ is denoted by $r_{\mathcal{H}}$.

Lemma 4.1. Let $H<W$ be a finitely generated subgroup. Suppose that for any $w \in H$ and any $w$-essential half-space $\mathcal{H}$, the reflection $r_{\mathcal{H}}$ belongs to $H$. Then $H$ contains a parabolic subgroup of $W$ as a normal subgroup of finite index.

Proof. Let $P<H$ be the subgroup of $H$ generated by all reflections $r_{\mathcal{H}}$ associated to a $w$-essential half-space $\mathcal{H}$ for some element $w \in H$. Thus $P$ is a reflection subgroup of $W$ contained in $H$. In particular $P$ is itself a Coxeter group, see Lemma 2.1.

A crucial point, which follows from [Kra09, Th. 5.8.1] and [CH06, Lem. 5.3], is that $W$ admits a finite index torsion-free subgroup $W^{\prime}$ such that for all $w \in$ $W^{\prime}$, we have

$$
\left.\operatorname{Pc}(w)=\left\langle r_{\mathcal{H}}\right| \mathcal{H} \text { is a } w \text {-essential half-space }\right\rangle .
$$

In particular $W^{\prime} \cap H$ is contained in $P$ and hence $P$ has finite index in $H$.

We now choose $w \in P$ in such a way that in the Coxeter group $P$, the parabolic closure $\operatorname{Pc}_{P}(w)$ of $w$ relative to $P$ is the whole $P$. Such an element $w$ always exists, see [CF, Cor. 3.3]. Let also $P^{\prime}$ denote the parabolic subgroup of $W$ generated by all those reflections $r_{\mathcal{H}}$ such that $\mathcal{H}$ is a $w$-essential half-space. By the definition of $P$, we have $P^{\prime} \subset P$. By the property recalled in the preceding 



(iii) For each minimal hyperbolic $J \subset S$, the set $J^{\perp}$ is spherical.

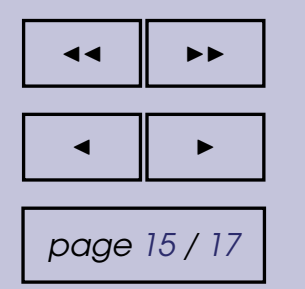

go back

full screen

close

quit

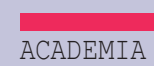

PRESS

NU

$\underline{\underline{\underline{I I I I I}}}$

UNIVERSTEIT

GENT

Proof. The main point is that, given a Coxeter system $(W, S)$, it is well known the group $W$ is virtually abelian if and only if it is a direct product of finite and affine Coxeter groups, i.e. if $S$ is Euclidean (see e.g. [MV00]).

(i) $\Rightarrow$ (iii).

Let $\mathscr{T}$ be a collection of subsets of $S$ satisfying (RH1) and (RH2). If (iii) fails, then there exist a minimal hyperbolic subset $J$ and a non-spherical irreducible subset $I \subset J^{\perp}$. By (RH1) there exists $K \in \mathscr{T}$ such that $I \cup J \subset K$. Then $\langle K\rangle$ is not virtually abelian since it contains $\langle J\rangle$, hence (i) fails as well.

(iii) $\Leftrightarrow$ (ii).

If (ii) fails then there exists a non-spherical and non-affine subset $J \subset S$ such that $J^{\perp}$ is non-spherical. Now any minimal non-spherical and non-affine subset $I$ of $J$ is minimal hyperbolic, and since $I \subset J$ we have $I^{\perp} \supset J^{\perp}$. Thus (iii) fails as well.

(ii) $\Rightarrow$ (i).

The condition (ii) clearly implies that for each irreducible non-spherical subset $J$, either $J$ is affine and $J \cup J^{\perp}$ is Euclidean or $J$ is non-affine and $J^{\perp}$ is spherical. In particular, every irreducible affine subset is contained in a unique maximal Euclidean subset. In other words the collection $\mathscr{T}$ of maximal Euclidean subsets of $S$ satisfies (RH2). Moreover (RH1) clearly holds as well.

Proof of Corollary D. In view of Theorem A, Lemma 4.2 and [HK05, Th. 1.2.1], it is enough to prove that $W$ is relatively hyperbolic with respect to its maximal virtually abelian subgroups if and only if it is relatively hyperbolic with respect to its maximal parabolic subgroups of Euclidean type. Since any parabolic subgroup of Euclidean type is virtually abelian, the 'if' part is clear. Conversely, assume that $W$ is relatively hyperbolic with respect to its maximal virtually abelian subgroups. Then conditions (i) and (ii) hold. In view of [CM05, Prop. 3.2], this implies that the parabolic closure of any virtually abelian subgroup of rank $\geq 2$ is of Euclidean type. In particular, if $A<W$ is a maximal virtually abelian subgroup, then $A=\operatorname{Pc}(A)$.

Proof of Corollary E. Assertions (i) and (iii) follow from [HK05, Theorem A.0.1]. For (iv), one applies [DS05, Proposition 5.4]; one needs the fact that any asymptotic cone of a Euclidean building is itself a Euclidean building: this is established in [KL97, Theorem 1.2.1]. The fact that $W$ is biautomatic follows from 
[HK05, Theorem 1.2.2(5)]. The biautomaticity of $\Gamma$ can then be deduced either directly from [Swi06] or from (i) together with [Reb01] and the fact that cocompact lattices of Euclidean buildings are biautomatic by [Swi06].

\section{References}

[BH93] B. Brink and R. B. Howlett, A finiteness property and an automatic structure for Coxeter groups, Math. Ann. 296 (1993), 179-190.

[Bou68] N. Bourbaki, Éléments de mathématique. Fasc. XXXIV. Groupes et algèbres de Lie. Chapitre IV: Groupes de Coxeter et systèmes de Tits. Chapitre V: Groupes engendrés par des réflexions. Chapitre VI: systèmes de racines. Actualités Scientifiques et Industrielles, No. 1337, Hermann, Paris, 1968.

[Bou97] M. Bourdon, Immeubles hyperboliques, dimension conforme et rigidité de Mostow, Geom. Funct. Anal. 7 (1997), 245-268.

[Bow99] B. H. Bowditch, Relatively hyperbolic groups, preprint, available at http://eprints.soton.ac.uk/29769/01/bhb-relhyp.pdf, 1999.

[Cap06] P.-E. Caprace, Conjugacy of 2-spherical subgroups of Coxeter groups and parallel walls, Algebr. Geom. Topol. 6 (2006), 1987-2029.

[CF] P.-E. Caprace and K. Fujiwara, Rank-one isometries of buildings and quasi-morphisms of Kac-Moody groups, Geom. Funct. Anal. 19 (2010), 1296-1319.

[CH06] P.-E. Caprace and F. Haglund, On geometric flats in the CAT $(0)$ realization of Coxeter groups and Tits buildings, Canad. J. Math. 61 (2009), 740-761.

[CM05] P.-E. Caprace and B. Mühlherr, Reflection triangles in Coxeter groups and biautomaticity, J. Group Theory 8 (2005), 467-489.

[Dav98] M. Davis, Buildings are CAT(0), in Geometry and cohomology in group theory (Durham, 1994) (Cambridge) (H. Kropholler et al., ed.), London Math. Soc. Lecture Note Ser. 252, Cambridge Univ. Press, 1998, pp. 108-123.

[Deo82] V. V. Deodhar, On the root system of a Coxeter group, Comm. Algebra 10 (1982), 611-630. 
